Kampiatu and Cozean Afr. J. Infect. Dis. (2015) 9(1): 6 - 9

\title{
A CONTROLLED, CROSSOVER STUDY OF A PERSISTENT ANTISEPTIC TO REDUCE HOSPITAL-ACQUIRED INFECTION
}

\author{
Patrick Kampiatu and Jesse Cozean
}

\author{
PCEA Kikuyu Hospital, Kenya; 45 Hospital Rd, Kikuyu, Kenya \\ E-mail: jcozean@gmail.com
}

\begin{abstract}
Background: Alcohol sanitizers have not been demonstrated to reduce hospital-acquired infections over handwashing alone in controlled, welldesigned clinical studies. A major reason for this may be the failure of traditional alcohol sanitizers to provide any residual, or persistent, activity. Any lapse in hand hygiene protocol then leaves patients and staff open to transmitting pathogens. The purpose of the study was to determine whether a persistent sanitizer would reduce hospital-acquired infection in a hospital setting as compared to handwashing alone.

Materials and Methods: A controlled, crossover study was conducted in three wards of a hospital over the course of three months. Two months were assigned as control periods, while the third month the test product was added. More than 6,000 patient-days were evaluated. Hospital staff were given a questionnaire to determine overall satisfaction with the product. The product used was a persistent, alcohol-based hand sanitizer (Zylast Antiseptic, $76 \% \mathrm{v} / \mathrm{v}$ ethyl alcohol)

Results: Control data showed that the hospital had a standard hospital-acquired infection rate of $23.1 \%$ across the three wards. No hospital-acquired infections were reported during use of the Antiseptic. The product was well tolerated by the staff and improved skin condition.

Conclusion: The Antiseptic was demonstrated to significantly $(\mathrm{p}<0.0005)$ reduce the hospital-acquired infection rate as compared to handwashing alone and is well-tolerated in a healthcare setting. The novel, persistent product has the ability to significantly reduce hospital-acquired infection and improve patient care in medical facilities.
\end{abstract}

Key words: Hospital-acquired infection, benzethonium chloride, antiseptic, Zylast, nosocomial infection, persistent, hand hygiene

\section{Introduction}

Hospital-acquired infections (HAIs) are a serious danger to patients, particularly as a growing percentage of these infections are resistant to antibiotics (Hawkey and Jones, 2009). Between five and ten percent of patients admitted will acquire an infection while in the hospital (Centers for Disease Control and Prevention, 2002). The increased resistance to antibiotics has made treatment costs even higher; HAIs cause nearly 100,000 deaths annually in the United States, (Centers for Disease Control and Prevention, 2002) as well as 10\% of admitted patients in developing countries (World Health Organization, 2013).

Much of the spread of nosocomial infection in hospitals is linked to a lack of compliance in hand hygiene. While the United States Centers for Disease Control (CDC) recommends handwashing for 1-2 minutes, multiple studies have shown that the actual duration among heathcare workers is often less than 15 seconds (Quraishi, McGuckin and Blals, 1984). Even with the presence of alcohol-based sanitizers in convenient locations near patient rooms and in hallways, compliance with hand hygiene is only 40\% (Erasmus, 2010). Skin irritation from repeated washing or the drying of the skin with traditional alcohol-based sanitizers may play a large role in the failure to comply with hand hygiene protocol among healthcare workers, as nearly $85 \%$ of healthcare workers have reported dry, cracked, or irritated skin as the result of hand hygiene products (Centers for Disease Control and Prevention, 2002).

However, improvements in compliance with hand hygiene protocols do not necessarily result in a reduction in the rate of HAIs in hospitals. In one study, compliance improved from $38 \%$ to $69 \%$ as a result of introducing a hand sanitizer in addition to handwashing alone, but the authors concluded, 'This improvement in the hand hygiene adherence rate was not associated with detectable changes in the incidence of healthcare-associated infection' (Rupp, 2008). Another study monitored hand hygiene and pathogen transmission, concluding there was "no correlation between the transmission rates of nosocomial pathogens... and hand hygiene compliance" (Eckmans, Schwab et. al, 2006).

A major limitation of traditional alcohol-based sanitizers is the inability to provide persistent protection against transient germs; once the product evaporates, antimicrobial activity no longer exists (Centers for Disease Control and Prevention, 2002). In other words, hands can immediately become recontaminated when they come into contact with a surface containing bacteria or viruses, leaving patients unprotected when healthcare workers do not adhere to hand hygiene protocol. The United States Food and Drug Administration states in their Tentative Final Monograph, 'Persistence, defined as prolonged activity, is a valuable attribute that assures antimicrobial activity during the interval between washings, and is important to a safe and effective healthcare personnel handwash' (FDA, 1994).

For these reasons, multiple clinical studies have proven that the addition of a traditional, alcohol-based hand sanitizer does not reduce infection rates in hospital settings when compared to handwashing alone (Rupp et al, 2008; Parienti et al, 2002) and may increase the risk of infection for patients by up to 33.4\% (Doebbeling et al, 1992; Larsen et al, 2005). Another study in long-term care facilities showed that facilities that relied on a traditional alcohol sanitizer were six times more likely to have a disease outbreak than ones that rely on handwashing alone (Blaney et al, 2011).

The purpose of this clinical study is to examine the ability of a novel hand sanitizer with persistent antimicrobial activity against transient bacteria to reduce the rate of infection in a hospital setting. The novel antiseptic product used in this trial has demonstrated rapid, broad-spectrum kill in the FDA-specified time-kill testing, showing an average reduction of $>99.999 \%$ within 15 seconds of the 25 different microorganisms tested (Czerwinski et al, 2014). The active ingredients have been shown to persistently pass the Surgical Scrub test as well as the Healthcare Personnel Handwash Test as described in the FDA Tentative Final Monograph (Shintre et al, 2006). After being applied to human skin and allowed to dry in the in-vivo Cup Scrub test, the Antiseptic destroyed more than $99.9 \%$ of a transient pathogen (E. coli) on contact at 20 minutes, 1 hour, and 6 hours after application (BioScience Laboratories, 2014). In contrast, a standard alcohol sanitizer had no effect at any of the timepoints tested. The test product 
http://dx.doi.org/10.4314/ajid.v9i1.2

has also been shown to be more than 100 times as effective in reducing the Norovirus surrogate on the skin than alcohol alone (Czerwinski and Cozean, 2012).

\section{Materials and Methods Study Design}

It was hypothesized that a product with rapid, broad spectrum kill that provides persistent protection and reduces irritation would reduce infection rates. The study tracked infection rates in three different wards of the hospital over 3 months. The first and third months served as controls, using the standard handwashing techniques of the hospital, while the novel antiseptic product was used in all three wards during the second month, allowing for a comparison of the rates of infection using the sanitizer versus standard handwashing techniques. This crossover design allowed each ward to serve as their own control, ensuring that differences in the wards or study populations did not affect the outcome of the study.

The control and treatment groups were displaced in time in accordance with recommendations from the Food \& Drug Administration. Because of the persistent efficacy of the Antiseptic, a nurse who used the persistent product and moved to another patient or ward would still have protection and would confound the study. The only way to perform a controlled study with a persistent product was to have the patients in the control and treatment groups completely displaced in time and/or space. There was no nursing crossover between the different wards of the study.

Each medical personnel entering a ward would perform one of the following tasks:

1) Wash his/her hands according to standard hand hygiene protocols as part of the control group or;

2) Apply Antiseptic per the instructions on the bottle as part of the treatment group. The instructions state, "Wet hands thoroughly with product and allow to dry without wiping."

No additional training, education, reminders, or materials were given to the experimental group.

Facility

The hospital selected for this clinical study was Kikuyu Hospital, one of the foremost private hospitals in East Africa. Three wards were used in this trial to evaluate the Antiseptic: the Men's Ward, the Women's Ward, and the Eye Care Ward. The staffs of the three wards were separate, and there was minimal staff or patient interaction between the wards.

\section{Test Product}

The active ingredient in the Zylast Antiseptic (manufactured by Innovative BioDefense, Inc.) used in this trial is $76 \%$ ethanol. Persistence is provided by a synergistic combination of ingredients centered around benzethonium chloride (BZT), a compound which has been known since the 1970 's to be safe and effective against both bacteria and viruses and has a persistent effect. The efficacy is enhanced by a proprietary, patent-pending combination of ingredients that reduces irritation to the skin, enhances efficacy against gram-negative bacteria, and allows persistent efficacy against transient germs for six hours. Like alcohol, the product kills all test strains of bacteria to a $99.99 \%$ level in seconds. The manufacturer suggests a dosage amount of $0.4 \mathrm{ml}$ per use, which was regulated by the dispenser.

\section{Data Collection}

Data was collected over the course of three months in each of the wards. Data collection began with an assessment of the rate of infection with standard handwashing techniques in the three wards. The following month, the Antiseptic was cycled through the three wards, beginning in July. Data were collected on all patients. Infections that were present upon admittance were noted and recorded, and any new HAI was recorded daily. The antiseptic product was then removed from the wards and data were collected for an additional month to ensure the accuracy of the control data. For ease of data collection in an overcrowded hospital, the data taken were not as a percentage of patients that presented with an HAI (patients who acquire infections / total number of patients), but rather as a percentage of the total occupied beds that had patients with HAIs on a given day (number of patients in hospital bed with HAI that day / total number of patients in hospital beds that day). While those numbers are directly proportional, they are not equivalent. Because acquiring a HAI increases the chances that a patient will spend more time in a hospital bed than a patient without a nosocomial infection, the method used in this study gives a higher number than percentage of patients who acquire an HAI. However, these methods are consistent between the control and treatment groups, and any comparison between the two groups remains valid.

Two outcome measures were monitored:

1) Rate of Infection: Each day, the number of patients in the ward were counted. Of those patients, those with infections that were not present when they entered the hospital were recorded, which represented the daily rate of patients with HAIs (Table 1).

2) Staff Survey: At the end of the trial, staff members were asked a series of questions regarding their experiences with the Antiseptic. Any skin effects were noted. Their opinions of the ease of use and efficacy of the new hygiene program were also recorded.

\section{Statistical Analysis}

Pearson's Chi-Squared Test for independence was used to analyze the data for statistical significance. The hypothesis tested in this crossover study was that HAIs would decrease in the treatment group using Zylast over the control group using hand-washing (n=6772). The level of significance was established at $\mathrm{p}<0.05$.

\section{Results}

The control data showed high rates of HAIs in all three wards. Over 6,000 patient-days were recorded in the study. Of these data points, 2,256 were part of the treatment group. With the addition of the Antiseptic, no hospital-acquired infections were reported in any of the three wards 
http://dx.doi.org/10.4314/ajid.v9i1.2

during the month when the product was installed and used (Table 1). When the hospital returned to hand-washing and removed the Antiseptic, the wards returned to their prior rates of infection.

The Questionnaire filled out by members of the hospital staff showed that the product was well-tolerated, improved skin condition and compliance with hand hygiene protocols, and was recommended by both the administration and healthcare staff. The Matron of the hospital summarized staff responses below.

The staff reported using the sanitizer much more often than they would have washed their hands. Of the 26 nurses that reported, none reported any skin irritation or adverse events. All remarked on the improvement of the skin as compared to washing with soap and water, and the staff universally recommended the adoption of the Antiseptic.

Table 1: Patient-Day Reporting

\begin{tabular}{|l|c|c|c|c|}
\hline & Control Patient-Days & Control HAIs & Treatment Patient-Days & Treatment HAIs \\
\hline Men's Ward & 1665 & $563(33.8 \%)$ & 858 & $0(0 \%)$ \\
\hline Female Ward & 1471 & $150(10.2 \%)$ & 738 & $0(0 \%)$ \\
\hline Eye Ward & 1377 & $329(23.9 \%)$ & 663 & $0(0 \%)$ \\
\hline Total & 4513 & $1042(23.1 \%)$ & 2259 & 0 \\
\hline
\end{tabular}

The primary outcome measurement, the presence of an HAI, is a categorical measurement and suitable for the chi-squared test. The calculated chi-squared statistic is 376.8 with two degrees of freedom, resulting in a p-value of $<0.0005$. Statistical analysis indicates the reduction in HAIs seen in this study was highly significant.

The Questionnaire filled out by members of the hospital staff showed that the product was well-tolerated, improved skin condition and compliance with hand hygiene protocols, and was recommended by both the administration and healthcare staff. The Matron of the hospital summarized staff responses below.

The staff reported using the sanitizer much more often than they would have washed their hands. Of the 26 nurses that reported, none reported any skin irritation or adverse events. All remarked on the improvement of the skin as compared to washing with soap and water, and the staff universally recommended the adoption of the Antiseptic.

Table 2: Staff Questionnaire

\begin{tabular}{|l|c|c|}
\hline \multicolumn{1}{|c|}{ Question } & \multicolumn{1}{c|}{$\begin{array}{c}\text { Options } \\
\text { (Circle One) }\end{array}$} & Staff Response \\
\hline $\begin{array}{l}\text { Do you feel that the availability of a hand sanitizer improved } \\
\text { compliance with hand hygiene protocols? }\end{array}$ & Yes/No & Better \\
\hline $\begin{array}{l}\text { Did your hands feel better, worse, or the same after using the } \\
\text { Antiseptic for one month instead of the traditional handwashing? }\end{array}$ & Better/Worse/The Same & Yes \\
\hline $\begin{array}{l}\text { Would you recommend the permanent addition of the Antiseptic from } \\
\text { this trial to the hospital? }\end{array}$ & Yes/No & \\
\hline
\end{tabular}

\section{Discussion}

The addition of the novel antiseptic product with persistence has been demonstrated to significantly $(\mathrm{p}<0.0005)$ reduce hospital-acquired infection in a hospital study of more than 6,000 patients over three months when compared to handwashing alone. It was well-tolerated by the hospital staff and improved skin condition. The hospital staff noted the presence of the Antiseptic increased compliance with hand hygiene protocol and plan to continue use in their hospital. The benefit can be attributed to the persistent efficacy of the product and the improvement in compliance with reduced skin irritation. Additional clinical studies are recommended to confirm the results seen in this study. No additional training or monitoring was necessary for the improvement, indicating that similar improvement could be seen in other facilities that implement hand hygiene products with persistent efficacy.

In their Hand Hygiene Guidelines, the CDC recommended research be conducted on persistent antibacterial products to "determine if preparations with persistent antimicrobial activity reduce infection rates more effectively than do preparations whose activity is limited to immediate effect" (Center for Disease Control, 2002). This study demonstrates it is possible to reduce nosocomial infections simply by the addition of a persistent sanitizer, the first time a sanitizer has showed a reduction in HAIs in a large well-controlled, crossover study. In conclusion, a persistent product was shown to be superior to products whose activity is limited to immediate effect in reducing infection rates and preventing HAIs.

Competing Interest: The antimicrobial test product for this trial was provided by the manufacturer, Innovative BioDefense Inc. No other financial support was provided. All data was independently compiled and reported by Kikuyu Hospital. 
Kampiatu and Cozean Afr. J. Infect. Dis. (2015) 9(1): 6 - 9

http://dx.doi.org/10.4314/ajid.v9i1.2

\section{Acknowledgement}

We gratefully acknowledge the physicians, nurses, and staff of Kikuyu Hospital who participated in this study.

\section{References}

1. BioScience Laboratories, Clinical evaluation of the persistent antimicrobial efficacy of two test products and one control product based on ASTM E2752-101, 1/14/2014

2. Blaney $\mathrm{D}$ et al. Use of alcohol-based hand sanitizers as a risk factor for norovirus outbreaks in long-term care facilities in northern New England: December 2006 to March 2007, American Journal of Infection Control. 2011; 39 (4) 296-301 [http://dx.doi.org/10.1016/j.ajic.2010.10.010]

3. Centers for Disease Control and Prevention, Estimating Health Care-Associated Infections and Deaths in U.S. Hospitals, 2002, Public Health Reports. 2007; 122

4. Czerwinski SE, Cozean J, Cozean C, Novel water-based Antiseptic Lotion compared with Alcohol Sanitizer Demonstrates Rapid, BroadSpectrum Kill, Journal of Infection and Public Health (2014) 7, 199-204

5. Czerwinski S, Cozean J. An Evaluation of a Hand Sanitiser Product to Reduce Norovirus Cross Infection, British Global and Travel Health Association. 2012; 20:42-46

6. Doebbeling BN, Stanley GL, Sheetz CT, et al. Comparative efficacy of alternative handwashing agents in reducing nosocomial infections in intensive care units, New England Journal of Medicine, 1992; 327: 88-93 [http://dx.doi.org/10.1056/NEJM199207093270205]

7. Eckmans T, Schwab F, et al., Hand rub consumption and hand hygiene compliance are not indicators of pathogen transmission in intensive care units. Journal of Hospital Infection, 63(4): 406-11

8. Erasmus V et al. Systematic Review of Studies on Compliance with Hand Hygiene Guidelines in Hospital Care, Infection Control and Hospital Epidemiology. 2010; 31(3) [http://dx.doi.org/10.1086/650451]

9. Hawkey PM, Jones AM. The changing epidemiology of resistance. The Journal of Antimicrobial Chemotherapy. 2009; 64 S1: i3-10 [http://dx.doi.org/10.1093/jac/dkp256]

10. Larson EL, Cimiotti J, Haas J, et al. Effect of antiseptic handwashing vs alcohol sanitizer on health care-associated infections in neonatal intensive care units. Arch Pediatr Adolesc Med. 2005; 159(4): 377-83 [http://dx.doi.org/10.1001/archpedi.159.4.377]

11. Parienti JJ et al. Hand-rubbing with an aqueous alcoholic solution vs. traditional surgical hand-scrubbing and 30-day surgical site infection rates: a randomized equivalence study, JAMA, 2002; 288:722-727 [http://dx.doi.org/10.1001/jama.288.6.722]

12. Rupp M et al. Prospective, Controlled, Cross - Over Trial of Alcohol - Based Hand Gel in Critical Care Units, Infection Control and Hospital Epidemiology. 2008; 29:1 [http://dx.doi.org/10.1086/524333]

13. Shintre, M., Gaonkar, T. Modak S. Efficacy of an alcohol-based healthcare hand rub containing synergistic combination of farnesol and benzethonium chloride, International Journal of Hygiene and Environmental Health. 2006; 209: 477-487 [http://dx.doi.org/10.1016/j.ijheh.2006.04.006]

14. World Health Association. Health Care Associated Infections: Fact Sheet. http://www.who.int/gpsc/country_work/gpsc_ccisc_fact_sheet_en.pdf; $31 / 10 / 2013$

15. Quraishi Z, McGuckin, Blals F. Duration of handwashing in intensive care units: A descriptive study, American Journal of Infection Control, American Journal of Infection Control. 1984; 12:2, 83-87 [http://dx.doi.org/10.1016/0196-6553(84)90021-X] 